\title{
Mehr Magenkrebs durch Alkohol - aber nicht bei jedem!
}

\begin{abstract}
Alkohol fördert die Entstehung von Magenkarzinomen. Der Effekt scheint sich aber nur bei Menschen bemerkbar zu machen, die nicht mit Helicobacter pylori infiziert sind.
\end{abstract}

Zwei Metaanalysen haben den Konsum von Alkohol als Risikofaktor für Magenkrebs ausgewiesen. Trotzdem ist der Zusammenhang umstritten: Die International Agency for Research on Cancer (IARC) hat Widersprüche in den publizierten Daten festgestellt. Einer Studie aus Korea zufolge könnten die unterschiedlichen Ergebnisse mit dem Helicobacter (H.)-pylori-Status zusammenhängen. Nur bei Teilnehmern ohne den Magenkeim war starker Alkoholkonsum mit einer erhöhten Magenkrebsrate assoziiert.

In der Gesamtkohorte mit 18.863 Probanden, darunter 403 Magenkarzinom- patienten, war das Risiko für den Krebs signifikant erhöht, wenn Alkohol seit mehr als 30 Jahren, mindestens siebenmal pro Woche oder in einer Menge von mindestens $55 \mathrm{~g}$ bei einer einzelnen Gelegenheit (Binge-Drinking) konsumiert wurde.

\section{Wie ist der Zusammenhang?}

Für 949 Personen, einschließlich 266 an Magenkrebs erkrankten, waren Daten zum H.-pylori-Status vorhanden. Berücksichtigte man diese Information sowie Alter, Geschlecht, BMI, Bildungsniveau und Rauchstatus der Teilnehmer, waren die Assoziationen zwischen Alkoholkonsum und Krebsraten nicht mehr signifikant. Nur Teilnehmer, die keine H.-pylori-spezifischen IgG-Antikörper aufwiesen, steigerten durch starken Alkoholkonsum ihr Magenkrebsrisiko: Bei mindestens sieben alkoholischen Getränken pro Woche war das Risiko rund 3,5-mal und bei BingeDrinking rund 3,3-mal so hoch wie bei Alkoholabstinenz. Bei den Patienten mit IgG-Antikörpern, die mit H. pylori infiziert waren, zeigte sich keinerlei Korrelation zwischen Trinkverhalten und Krebsrate. Warum aber sollte Alkohol bei Menschen mit einer H.-pylori-Infektion weniger Schaden anrichten als bei nicht infizierten? Die Studienautoren um SeungHyun Ma vermuten antimikrobielle Effekte von Alkohol auf den Magenkeim. Hinweise auf eine solche Wirkung hätten sich auch in anderen epidemiologischen Studien ergeben. (BS)

MaSH et al. BJC 2015; online 17. September. doi: 10.1038/bjc.2015.333

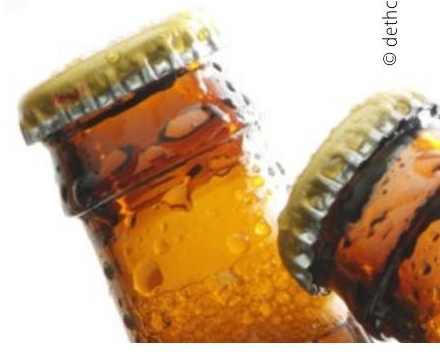

nicht mehr als Standard festgelegt wurde, bleibt abzuwarten, ob nicht doch bei schwer zu behandelnden Patienten mit Genotyp 3 (vorbehandelt mit fortgeschrittener Leberfibrose/Zirrhose) eine Triple-Therapie effektiver und kostengünstiger ist.

\section{Ist SVR12 ein sicherer Marker für die Ausheilung einer Hepatitis C?}

Zahlreiche interferonfreie Therapieregime sind seit 2014 für die Behandlung der chronischen Hepatitis C zugelassen worden. Inzwischen wird die Ausheilung einer chronischen Hepatitis C regelhaft 12 Wochen nach Therapieende (SVR12) im Gegensatz zur interferonbasierten Therapie (SVR24) dokumentiert. Inzwischen liegen insbesondere für Sofosbuvir Daten von mehr als 3000 Patienten aus Phase-IIII-Studien mit einer SVR12 vor [10]. Ein später Relapse mehr als 12 Wochen nach Therapieende trat nur bei $0,17 \%$ der Patienten auf. Die meisten Virämien, die mehr als 12 Wochen nach Therapieende auftraten, waren auf Neuinfektionen zurückzuführen. Somit kann mit sehr hoher Wahr- scheinlichkeit für eine Sofosbuvir-basierte Therapie eine Ausheilung der Hepatitis C bereits 12 Wochen nach Therapieende vorhergesagt werden.

\section{Praxisrelevanz \& Fazit}

Nach der Evolution der interferonfreien Therapie der chronischen Hepatitis C im vergangenen Jahr ist inzwischen eine Konsolidierung eingetreten. Nahezu alle zugelassenen interferonfreien Therapieregime weisen extrem hohe Heilungsraten bei guter Verträglichkeit und kurzer Therapiedauer auf. Der ,einfache Hepatitis CPatient" kann mit über 90\%-iger Wahrscheinlichkeit geheilt werden. Daher fokussiert sich inzwischen die Forschung auf spezielle, schwer zu behandelnde $\mathrm{Pa}$ tientengruppen. Patienten mit HCV-Reinfektion nach Transplantation oder HCV-HIV-koinfizierte Patienten gehören hier kaum noch dazu, da sich die Ansprechraten für diese Gruppen denen der Monoinfizierten angleichen. Bleiben $\mathrm{Pa}$ tienten mit fortgeschrittener/dekompensierter Leberzirrhose, Patienten mit Niereninsuffizienz oder seit Kurzem auch $\mathrm{Pa}$ - tienten mit einem Therapieversagen (Relapse) auf eine interferonfreie Ersttherapie. Auch für diese Patientengruppen scheinen erfolgversprechende Therapieregime zur Verfügung zu stehen. Beim HCV-Genotyp 3, dem am schwersten zu behandelndem Genotyp, stellt sich nach dem diesjährigen europäischen Leberkongress die Frage, ob nicht doch eine 12-wöchige interferonbasierte Triple-Therapie insbesondere bei fortgeschrittener Leberfibrose durchgeführt werden sollte, auch wenn nach G-BA-Beschluss für diese Therapie kein Zusatznutzen besteht. Dies weist erneut nur auf das Dilemma der neuen wissenschaftlichen Erkenntnisse und den gleichzeitigen Beschränkungen in der praktischen Anwendung (Zulassung, GBA-Nutzenbewertung, Kosten) hin. Insgesamt hat sich die Datenbasis zur interferonfreien Therapie jedoch weiter vergröBert, so dass sich hieraus für die Praxis eine zunehmende Therapiesicherheit für den individuellen Patienten mit chronischer Hepatitis C ergibt.

Literaturliste beim Verlag 